\title{
Ağ Úsáid
}

Thacar

\section{Sonraí}

Linked

Logainm

Rebecca Grant

Nuno Lopes

Eoghan Ó Carragáin

Catherine Ryan

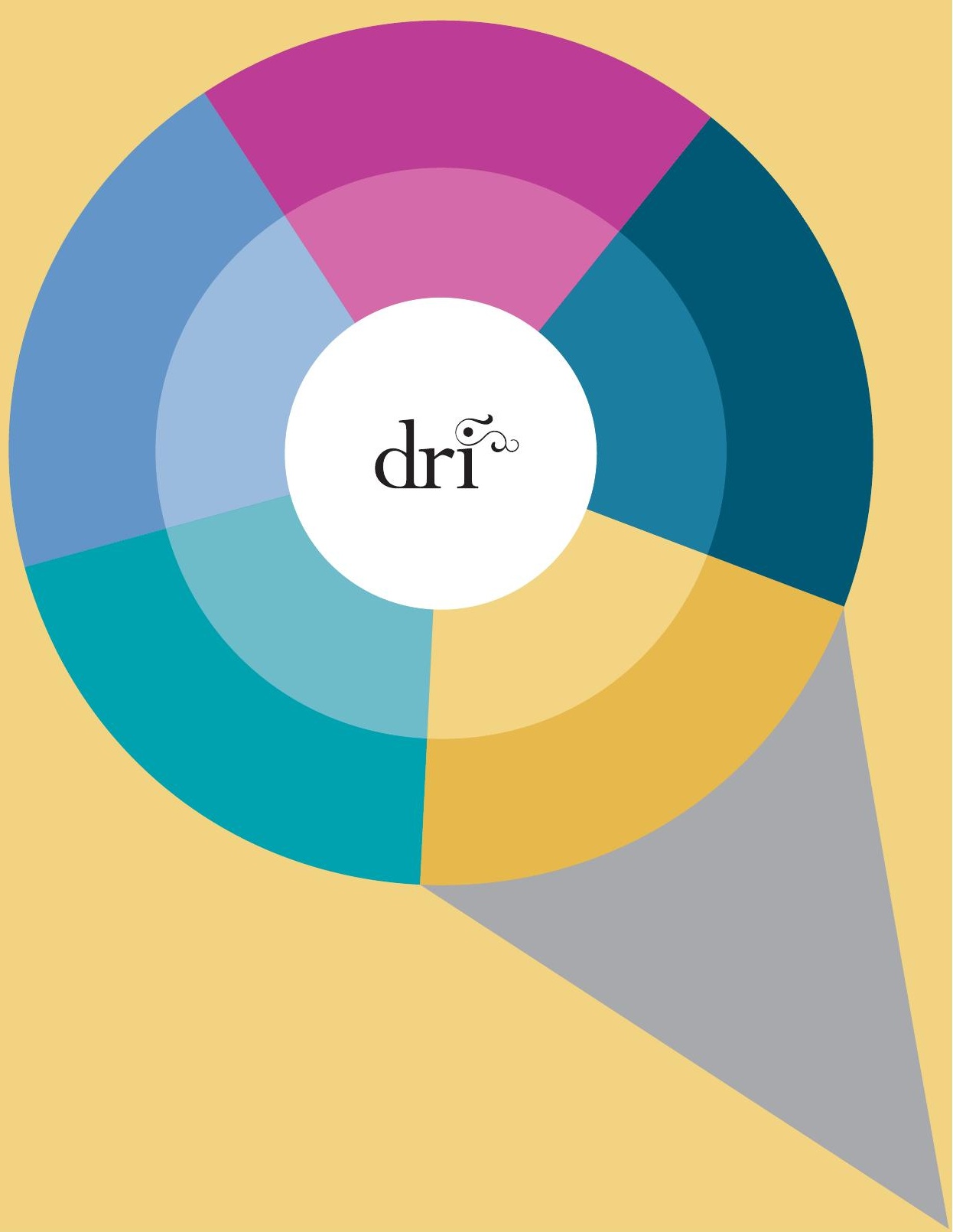




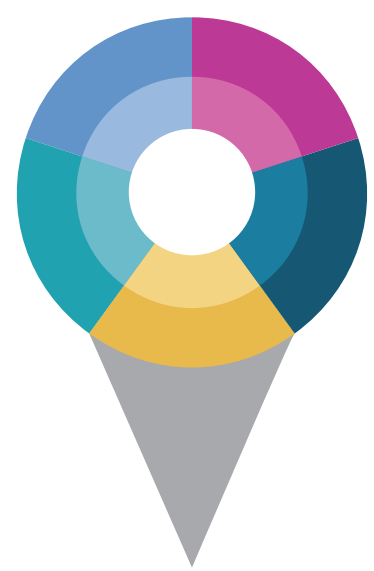

Is tionscadal comhoibríoch é Linked Logainm atá de chuid Taisclann Dhigiteach na hÉireann (DRI), an Institiúid um Thaighde ar an bhFiontraíocht Dhigiteach (DERI), Fiontar in Ollscoil Chathair Bhaile Átha Cliath agus Leabharlann Náisiúnta na hÉireann.

Sa tionscadal Linked Logainm cruthaíodh leagan le Sonraí Nasctha den bhunachar sonraí dátheangach údarásach de logainmneacha na hÉireann logainm.ie, atá á fhorbairt ag Fiontar i gcomhar leis an mBrainse Logainmneacha sa Roinn Ealaíon, Oidhreachta agus Gaeltachta.

Tá forléargas i dtuarascáil an tionscadail ar theicneolaíochtaí Sonraí Nasctha agus ar na próisis ba ghá chun tacar sonraí logainm.ie a thiontú ina Sonraí Nasctha agus chun iadsan a cheangal le tacair shonraí LinkedGeoData, Geonames agus DBpedia.

Tá réamheolas idirghníomhach ar an suíomh Gréasáin taispeána Location LODer ${ }^{1}$ maidir leis na féidearthachtaí a bhaineann leis an gcoincheap is bun le Linked Logainm. Is féidir le húsáideoirí féachaint ar ábhar ó fhoinsí éagsúla, Europeana, an Leabharlann Náisiúnta agus Wikipedia ina measc, trí úsáid a bhaint as sonraí logainm.ie agus comhéadan léarscáile.

Tá roinnt moltaí sa doiciméad seo faoi úsáidí eile a d'fhéadfaí a bhaint as tacar sonraí Linked Logainm.

1 http://apps.dri.ie/locationLODer 


\section{(c) (1)}

Tá an saothar seo ar fáil faoin gceadúnas Creative Commons Attribution $\mathbf{3 . 0}$ Ireland.

Úsáid an téacs seo a leanas agus an saothar seo á lua agat: Grant, R., Lopes, N., Ó Carragáin, E., Ryan, C. (2013), 'Ag usáid thacar sonraí Linked Logainm'. Baile Átha Cliath: Acadamh Ríoga na hÉireann agus Leabharlann Náisiúnta na hÉireann; Gaillimh: Ollscoil na hÉireann, Gaillimh.

DOI: 10.3318/DRI.LODer.2013.4

Aistriúchán: Fiontar in Ollscoil Chathair Bhaile Átha Cliath 


\section{Cé dóibh an doiciméad seo?}

Beidh tacar sonraí Linked Logainm úsáideach d'aon duine, d'aon tionscadal nó d'aon institiúid a mbeadh suim acu ábhar faoi logainmneacha na hÉireann a chur ar fáil ar an nGréasán. Sa mhéid gur foilsíodh sonraí logainm.ie i bhformáid struchtúrtha, atá inléite ag an ríomhaire, féadfaidh ríomheolaithe, forbróirí Gréasáin, an pobal oidhreachta agus gairmithe faisnéise eile úsáid a bhaint as na sonraí sin.

Cuirtear cásanna úsáide agus eiseamláirí arle fáil sa doiciméad seo dóibh siúd a bhfuil taithí theicniúil éigin acu agus ar suim leo leas a bhaint as tacar sonraí Linked Logainm. Roinneamar na heiseamláirí thíos ina dhá chuid mar seo - na heiseamláirí a bhaineann le hábhar do choimeádaithe sonraí agus do ghairmithe faisnéise, agus na heiseamláirí eile a bhaineann le hábhar do ríomheolaithe agus d'fhorbróirí.

\section{Coimeádaithe Sonraí agus Gairmithe Faisnéise}

Sna heiseamláirí thíos tá liosta d'uirlisí bogearraí ar nós Open Refine agus Pundit a bhfuil comhéadain shármhaithe acu don úsáideoir deiridh a chuireann ar chumas coimeádaithe sonraí agus gairmithe faisnéise oibriú go díreach le sonraí ó Linked Logainm (ar a dtugtar "Uirlisí don Úsáideoir Deiridh" thíos).

\section{Ríomheolaithe, Forbróirí agus Foilsitheoirí Sonraí}

Chun teacht ar na sonraí go díreach, is féidir le ríomheolaithe agus le forbróirí úsáid a bhaint as Linked Logainm sa chás go bhfuil cur amach acu ar theicneolaíochtaí Linked Logainm, mar shampla SPARQL agus RDF (ar a dtugtar "Uirlisí don Fhorbróir" thíos). 


\section{Cad is Sonraí Nasctha ann?}

"Linked Data is to spreadsheets and databases what the Web of hypertext documents is to word processor files"

Is é an rud atá i gceist le Sonraí Nasctha sonraí atá foilsithe ar an nGréasán de réir shraith prionsabal a dearadh chun go mbainfí na constaicí atá sa bhealach ar stóir shonraí a nascadh trí chur leis na croítheicneolaíochtaí Gréasáin a bhfuil teist mhaith orthu:

Úsáid URlanna (Aitheantóirí Uathúla Acmhainne) mar ainmneacha ar rudaí (e.g. áiteanna, daoine, coincheapa)

- Úsáid URlanna HTTP ionas gur féidir le daoine na hainmneacha sin a chuardach (ar an gcaoi chéanna is a chuardaíonn siad leathanach Gréasáin ag úsáid seoltaí HTTP) - Caighdeáin Sonraí Nasctha (RDF, SPARQL) a úsáid chun faisnéis úsáideach a sholáthar nuair a chuardaíonn duine URI

- Naisc le URlanna eile a sholáthar ionas gur féidir le daoine teacht ar rudaí breise. ${ }^{3}$

Glacadh leis na prionsabail agus na teicneolaíochtaí seo (URlanna, RDF, HTTP, RDFS, OWL) go hidirnáisiúnta ar an gcleachtas is fearr chun sonraí a fhoilsiú ar an nGréasán oscailte agus chun gaol a léiriú idir na sonraí sin.

Táthar tar éis teicneolaíochtaí Sonraí Nasctha a chur i bhfeidhm in iliomad earnálacha. Baineann ollscoileanna úsáid as Sonraí Nasctha chun feabhas a chur ar theicneolaíocht an oideachais agus na foghlama agus baintear úsáid as Sonraí Nasctha in earnálacha an chúraim sláinte, an rialtais, an fhuinnimh, TF agus i ríomhsheirbhísí turasóireachta chun feabhas a chur ar chuardach, ar chomhtháthú sonraí, ar bhainistiú inneachair agus ar aimsiú. Bíonn institiúidí cultúrtha ar nós Europeana agus BBC ag brath ar Shonraí Nasctha chun feabhas a chur ar chatagóiriú inneachair agus ar chomhtháthú sonraí, agus chun feabhas a chur ar chuardach agus ar aimsiú inneachair. Féadtar teacht ar fhorléargas ar thacair shonraí éagsúla de Shonraí Nasctha, chomh maith lena miondealú de réir catagóire agus fearainn, ar http://lod-cloud.net/state/.

Le haghaidh tuilleadh eolais ar na prionsabail agus ar na teicneolaíochtaí is bun le Sonraí Nasctha, féach an tAguisín ag deireadh an doiciméid seo.

\footnotetext{
2 http://www.w3.org/wiki/LinkedData

${ }^{3}$ http://www.w3.org/Designlssues/LinkedData
} 


\section{Uirlisí don Úsáideoir Deiridh}

Trí shonraí a fhoilsiú ar an nGréasán oscailte féadfaidh daoine na sonraí sin a athúsáid ar bhealaí nach rabhthas ag súil leo. Is iad seo a leanas roinnt féidearthachtaí atá bunaithe ar an úsáid a baineadh as Sonraí Nasctha i gcomhthéacsanna eile.

\section{Stór Focal Rialaithe d'lonchur Sonraí}

Is foinse uathúil é tacar sonraí logainm.ie do na leaganacha caighdeánacha údaraithe de logainmneacha na hÉireann, idir Bhéarla agus Ghaeilge. Sa mhéid go bhfuil an tacar sonraí seo ar fáil anois i bhformáid struchtúrtha ar an nGréasán oscailte, d'fhéadfaí úsáid a bhaint as mar fhoinse chun aonáin logainmneacha de chuid na hÉireanna chruthú i dtacair shonraí logánta nó i dtaifid mheiteashonraí.

D'fhéadfaí úsáid a bhaint as na sonraí chun seirbhís chuardaigh a fhorbairt bunaithe ar thacar sonraí Linked Logainm ionas go bhféadfaí é a chomhtháthú le foirmeacha ionchurtha sonraí i bhfeidhmchláir logánta.

Mar shampla, is uirlis chomhoibríoch fhoinse oscailte taighde é Pundit ${ }^{4}$, trínar féidir le húsáideoirí "not only to comment, bookmark or tag web pages, but also to create semantically structured data while annotating". Is féidir Pundit a chumrú chun aonáin Sonraí Nasctha a aisghabháil ó chríochphointí éagsúla SPARQL, Linked Logainm san áireamh.

\section{Glanadh agus Feabhsú Sonraí Oidhreachta nó Meiteashonraí}

Is é ceann de na dúshláin coitianta a bhíonn le sárú agus tacair shonraí á gcomhtháthú ná sonraí scaipthe nó neamhchaighdeánacha a cheartú.

Mar is amhlaidh leis an eiseamláir d'lonchur Sonraí thuas, féadtar úsáid a bhaint as an leagan Sonraí Nasctha de thacar sonraí logainm.ie mar fhoinse a bhféadfaí logainmneacha de chuid na hÉireanni dtacair shonraí logánta a chur i gcomparáid go huathoibríoch léi, chun a chinntiú go bhfuil siad ag teacht leis na leaganacha caighdeánacha Béarla nó Gaeilge.

${ }^{4} \mathrm{http}: / /$ www.thepund.it 
Is féidir le forbróirí cód a scríobh chun rochtain dhíreach a fháil ar na sonraí, nó uirlisí saor in aisce a úsáid, mar shampla Open Refine ${ }^{5}$, a "thugann chun réitigh" foinsí sonraí logánta le hacmhainní Sonraí Nasctha cosúil le Linked Logainm.

Chun seirbhís réitigh RDF Logainm a chur le Open Refine, is gá d'úsáideoirí dul chuig 'RDF' > 'Add reconciliation service' > 'Based on SPARQL endpoint...', agus ansin an fhaisnéis seo a leanas a chur isteach:

Name: (ainm ar bith don tseirbhís)

Endpoint URL: http://data.logainm.ie/sparql

Type: Virtuoso

Label Properties: Also check 'foaf:name'

\section{Feabhsú Córas Cuardaiğh aguus Aimsiúcháin}

Trí nasc a chruthú idir sonraí agus Linked Logainm (féach eiseamláirí 3 agus 4 thuas) agus trí thagairt a choimeád de URI Linked Logainm, d'fhéadfadh córas áitiúil rochtain a fháil ar an bhfaisnéis uathúil atá á forbairt ag Fiontar agus ag an mBrainse Logainmneacha den Roinn Ealaíon, Oidhreachta agus Gaeltachta, lena n-áirítear:

- Faisnéis maidir le coibhéis leaganacha Béarla agus Gaeilge de logainmneacha na hÉireann.

- Faisnéis maidir le leaganacha malartacha, dímholta nó neamhdheimhnithe de logainmneacha Gaeilge.

- Faisnéis maidir leis an ngaol ordlathach idir contaetha, barúntachtaí, bailte fearainn, paróistí agus gnéithe eile in Éirinn.

D'fhéadfaí an fhaisnéis bhreise seo a úsáid mar bhonn cumhachtach eolais d'fheidhmchláir cuardaigh agus aimsiúcháin, chun moltaí cuardaigh a chur ar fáil d'úsáideoirí nó chun iad a atreorú bunaithe ar ghaolmhaireachtaí struchtúrtha a d'aimseofaí i dtacar sonraí Linked Logainm.

${ }^{5} \mathrm{http}$ ://openrefine.org 


\section{Uirlisí don Fhorbróir}

\section{Foilsiú faisnéise maidir le loğainmneacha na hÉireann: mol nasctha le haghaidh URlanna}

Chomh maith le foilsiú sonraí le URlanna, le HTTP agus le RDF, gríosaíonn prionsabail na Sonraí Nasctha foilsitheoirí lena sonraí a nascadh le URlanna ó thacair shonraí oscailte eile.

Bíonn a lán ama agus costais i gceist chun tacair shonraí a nascadh go beacht, fiú amháin le huirlisí uathoibrithe, agus mar sin ní foláir d'fhorbróirí bheith roghnaitheach. Go praiticiúil, tá tacair mhóra shonraí oscailte ar nós DBpedia ${ }^{6}$, Freebase ${ }^{7}$ agus Geonames ${ }^{8}$ anois ar na moil nasctha a úsáidtear sa Néal Sonraí Nasctha. Trí nasc aonair a chruthú leis na moil choiteanna seo, féadfaidh foilsitheoirí a sonraí a nascadh leis na céadta nó leis na mílte de thacair shonraí.

Is é seo an cur chuige a roghnaíodh don tionscadal Linked Logainm, inar nascadh sonraí logainm.ie le DBpedia agus Geonames, agus ar an gcaoi sin nascadh le gach tacar sonraí eile iad a thagraíonn do na URlanna céanna ó na foinsí sin.

Ceann de na cúiseanna ar cuireadh tionscadal Linked Logainm ar bun ná nach raibh logainmneacha na hÉireann ar fad, go háirithe ag leibhéal na barúntachta, an pharóiste, agus an bhaile fearainn, ar fáil i dtacair shonraí cosúil le Dbpedia agus Geonames. Mar sin tá Linked Logainm ina thargaid an-úsáideach agus tacair shonraí á bhfoilsiú a thagraíonn do logainmneacha na hÉireann ar an leibhéal gráinneach, sa mhéid gur mó seans go mbeidh na haonáin sin ar fáil ann. Gan amhras, mar gheall ar go bhfuil naisc cruthaithe cheana féin idir Linked Logainm agus DBpedia agus Geonames, gheobhaidh foilsitheoirí an t-eolas ó na naisc eile sin freisin sa chás go meaitseálfaidh siad acmhainní le Linked Logainm.

Ar an gcaoi seo, féadfaidh Linked Logainm feidhmiú mar mhol logánaithe le haghaidh URlanna logainmneacha na hÉireann agus mar gheata isteach sa Néal Sonraí Nasctha i gcoitinne.

Tá roinnt uirlisí ar fáil chun cuidiú le naisc a chruthú idir tacair shonraí éagsúla RDF, Silk ${ }^{9}$ agus LIMES ina measc. ${ }^{10}$ Chun tacar sonraí Linked Logainm a chruthú bhaineamar úsáid

${ }^{6} \mathrm{http}: / /$ wiki.dbpedia.org/About

${ }^{7} \mathrm{http}: / / \mathrm{www}$.freebase.com/

$8 \mathrm{http}: / /$ www.geonames.org/

${ }^{9} \mathrm{http}: / /$ wifo5-03.informatik.uni-mannheim.de/bizer/silk/

$10 \mathrm{http} / / /$ aksw.org/Projects/LIMES.html 
as creatlach Silk chun na naisc a aithint idir Linked Logainm agus DBpedia, LinkedGeoData agus GeoNames. Is féidir le bunachair shonraí thriaracha Sonraí Nasctha a fhoilsiú de réir a gcuid sonraí, Openlink Virtuoso mar shampla. ${ }^{11}$ Nó is féidir úsáid a bhaint as uirlisí eile, mar shampla Pubby, ${ }^{12}$ chun Sonraí Nasctha a fhoilsiú ar aon chríochphointe SPARQL atá ar fáil.

\section{larratais a dhéanamh maidir le logainmneacha na hÉireann}

Ón mbliain 2008 i leith, tá an suíomh gréasáin logainm.ie ina fhoinse shaibhir agus uathúilfaisnéise maidir le logainmneacha na hÉireann do thaighdeoirí. Trí amhshonraí logainm.ie a fhoilsiú mar Shonraí Nasctha, féadfaidh forbróirí rochtain a fháil anois ar theicneolaíochtaí agus ar uirlisí caighdeánacha Sonraí Nasctha chun na gaoil atá ann i dtacar sonraí Logainm a chur lena gcuid sonraí agus feidhmchlár féin d'fhonn iad a fheabhsú.

Is féidir rochtain a fháil ar shonraí struchtúrtha Linked Logainm ó bhunachar sonraí logainm.ie agus iarratas a dhéanamh orthu ag http://data.logainm.ie/sparql/, ag úsáid na teanga iarratais SPARQL. Is féidir faisnéis struchtúrtha maidir le gach logainm a aisghabháil i srathuithe éagsúla (e.g. XML, N3, JSON) trí rochtain a fháil ar an aitheantóir faoi seach tríd an idirbheartaíocht inneachair.

Cuirtear síos ar shonraí Linked Logainm le foclóirín NeoGeo, ${ }^{13}$ mar a sainítear gach logainm mar chineál "Gné" agus mar a ndéantar na comhordanáidí geografacha faoi seach a nascadh le chéile mar aonán ar leith trí airí na "geoiméadrachta".

Mar shampla, is é http://data.logainm.ie/place/1375542 an URI Linked Logainm a shainaithnionn Cathair Bhaile Átha Cliath agus is é http://data.logainm.ie/geometry/ 1375542 an URI dá comhordanáidí geografacha. Sanntar 'cineál' do gach logainm a bhaineann go sonrach le tacar sonraí logainm.ie (mar shampla barúntacht, baile, baile fearainn etc.). Déantar na logainmneacha a nascadh le gach áit trí úsáid a bhaint as an airí "foaf:name" agus déantar iad a anótáil leis an gclib theanga faoi seach ("en" nó "ga").

\section{Rochtain ar Shonraí Nasctha le SPARQL}

Déantar acmhainní faisnéise a gcuirtear síos orthu le RDF, agus arna gcur in iúl in RDFS, OWL, SKOS agus teangacha eile, a shábháil ar chórais bainistíochta bunachair shonraí agus tugtar 'bunachar sonraí triaracha' orthusan. Ar an gcaoi chéanna a n-úsáideann bunachair shonraí choibhneasta SQL chun iarratas a dhéanamh ar tháblaí i mbunachair shonraí, baineann bunachair shonraí thriaracha úsáid as SPARQL (Prótacal SPARQL agus Teanga larratais RDF) ${ }^{\mathbf{1 4}}$ chun iarratas a dhéanamh ar fhaisnéis atá stóráilte i bhformáid thriarach RDF agus í a aisghabháil.

11 http://virtuoso.openlinksw.com/

12 http://wifo5-03.informatik.uni-mannheim.de/pubby/

$13 \mathrm{http}: / / g e o v o c a b . o r g / d o c / n e o g e o /$

14 http://www.w3.org/TR/rdf-sparql-query/ 
Is pointí isteach iad críochphointí SPARQL chun iarratas a dhéanamh ar bhunachair shonraí thriaracha le hiarratais SPARQL, agus déantar iadsan a struchtúrú mar seo (leis na heiseamláirí ó chríochphointe SPARQL Linked Logainm (http://data.logainm.ie/sparql):

\section{Basic query structure}

select * where $\{$ ?s ?p ?o $\}$

San eiseamláir thuas, is é "select" an cineál iarratais atá á chur isteach againn. Cuireann an réiltín (*) in iúl go dteastaíonn uainn go dtaispeánfaí gach athraitheach dúinn. Seasann na míreanna idir lúibíní catacha don ainmní, don fhaisnéis agus don chuspóir.

\section{Select all subjects, predicates and objects where the name of the subject is Dublin [results]]}

select * where \{ ?s ?p ?o. ?s foaf:name "Dublin"@en\}

San eiseamláir thuas táimid ag déanamh iarratais ar gach ainmní, ar gach faisnéis agus ar gach cuspóir sa chás gurb é Dublin an cuspóir (i mBéarla).

\section{Select all place names of type "county" [results]}

select ?s where $\{$ ?s rdf:type <http://data.logainm.ie/category/CON> \}

\section{Select the Irish and English names of "Dublin" and its geographical coor- dinates [results]}

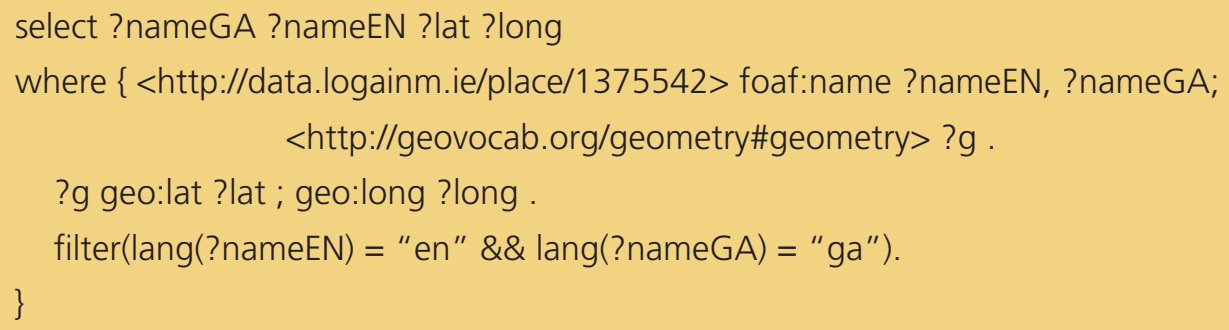

Select the URIs of "Dublin" in other data sets [results]

select ? uri where $\{<$ http://data.logainm.ie/place/1375542> owl:sameAs ?uri . \} Is féidir teacht ar ábhair teagaisc maidir leis an teanga SPARQL ag: 

http://learningsparql.com/
http://jena.apache.org/tutorials/sparql.html
- http://www.linkeddatatools.com/querying-semantic-data
- http://www.cambridgesemantics.com/semantic-university/sparql-by-example

\section{Rochtain ar Shonraí Nasctha trí thagairtí URlanna a bhaint}

Is féidir faisnéis struchtúrtha maidir le gach logainm a aisghabháil freisin, i bhformáidí éagsúla, ón aitheantóir faoi seach trí idirbheartaíocht inneachair. Is minic a úsáidtear CURL mar uirlis chun rochtain a fháil ar shonraí i mbealach atá neamhspleách ar theanga. ${ }^{15}$ Mar shampla, is é http://data.logainm.ie/place/1375542 an URI a shainaithníonn cathair Bhaile Átha Cliath agus is féidir úsáid a bhaint as curl chun rochtain a fháil ar na léiriúcháin éagsúla:

curl -H "Accept: text/rdf+n3" http://data.logainm.ie/place/1375542

Is iad seo a leanas na cineálacha éagsúla de Cheanntásca Accept HTTP:

'text/plain' le haghaidh léiriúchán NTriples

'text/rdf+n3' le haghaidh léiriúchán Turtle

'application/rdf+json' le haghaidh léiriúchán JSON

'application/rdf+xml' le haghaidh léiriúchán XML

Is féidir rochtain a fháil ar an bhfaisnéis seo freisin ó theangacha éagsúla, mar shampla, in Javascript is féidir na torthaí JSON a aisghabháil le JQuery:

\$.getJSON('http://data.logainm.ie/place/1375542', callbackFunction )

Ó Python is féidir torthaí comhchosúla a aisghabháil ag úsáid leabharlann na 'n-iarratas' (http://docs. python-requests.org/en/latest/):

import requests

requests.get("http://data.logainm.ie/place/1375542", headers = \{ 'Accept':

'application/rdf+json\})

${ }^{15} \mathrm{http}: / / c u r l . h a x x . s e /$ 


\section{Aģuisín: Gluais Téarmaí Sonraí Nasctha}

\section{URlanna}

Aitheantóirí Aonfhoirmeacha Acmhainne (URlanna) - baintear úsáid astu seo mar ainmneacha uathúla le haghaidh acmhainní ar an nGréasán. Baintear úsáid as URlanna chun ábhair, gaolmhaireachtaí, airíonna, luachanna nó aon rud eile atá le fáil ar an nGréasán a shainaithint, maille le 'fíor-nithe' cosúil le daoine agus áiteanna. Nuair a úsáidtear URI in ionad sraith téacs chun aonán nó acmhainn a shainaithint, ní bhíonn aon débhríocht ann idir daoine nó áiteanna a bhfuil an t-ainm céanna orthu, ní tharlaíonn earráidí le haonáin ainmniúcháin toisc go bhfuil focal mílitrithe nó toisc go bhfuil an phoncaíocht san áit mhícheart, e.g. faoi láthair tá roinnt córas bainistíochta leabharlainne a mheasfadh gur dhá aonán éagsúla iad 'James Joyce' agus 'James Joyce.' Ina ionad sin is féidir URI a úsáid mar aitheantóir le haghaidh James Joyce ${ }^{16}$.

\section{RDF}

Is é is Resource Description Framework (RDF) ${ }^{\mathbf{1 7}}$ ann ná samhail sonraí i ngraf arna forbairt ag W3C chun faisnéis a léiriú agus a mhalartú ar an nGréasán. Déanann RDF ráitis, ar a dtugtar 'sonraí triaracha', agus bíonn siadsan san fhoirm seo - ainmní, faisnéis agus cuspóir, agus is é an t-ainmní an t-aonán nó an acmhainn agus is é an cuspóir an acmhainn eile nó an luach. Is í an fhaisnéis an gaol eatarthu agus déantar í a shainiú le foclóiríní réamhshainithe. Trí chúpla ceann de na ráitis seo a chur le céile, cruthaítear líonra 'sonraí triaracha' (graf RDF). Leis an RDF is gá URlanna a úsáid chun

${ }^{16}$ Mar shampla an URI DBpedia http://dbpedia.org/resource/James_Joyce.

${ }^{17}$ http://www.w3.org/RDF/ 
rudaí agus gaolmhaireachtaí a ainmniú, agus ar an gcaoi seo, is féidir le ríomhairí na sonraí a thuiscint, bíonn na sonraí seasmhach, gan débhrí, agus is féidir iad a roinnt ar fud an Ghréasáin. Tá RDF srathaithe freisin i bhformáid XML agus i bhformáidí eile.

\section{Scéimre RDF}

Cé gurb é RDF an bhunsamhail sonraí is bun le Sonraí Nasctha agus leis an nGréasán Séimeantach, ní shainíonn sé na gaolmhaireachtaí idir na ráitis seo agus na téarmaí a úsáidtear chun iad a chur in iúl: úsáidtear teangacha eile chun sin a dhéanamh. Tá an Scéimire RDFS ar an teanga léirithe eolais is bunúsaí chun croíchóras cineáil a sholáthar d'aicmí agus d'airíonna agus cuireann sé in iúl an dóigh ar chóir iad go léir a úsáid le chéile. ${ }^{18}$

\section{Teanģa Ointeolaíochta Gréasáin (OWL)}

Is teanga léirithe eolais níos fairsinge agus níos léirithí é an Teanga Ointeolaíochta Gréasáin (OWL) ná RDFS agus baintear úsáid aisti go príomha mar theanga chun ointeolaíochtaí a chruthú agus a léiriú ar an nGréasán. Baintear úsáid aisti chun téarmaí a chur in iúl agus a shainiú i bhfearann is díol spéise nó in ábhar áirithe agus chun na gaolmhaireachtaí eatarthu a chur in iúl freisin.

Forbraíodh a lán ointeolaíochtaí le RDFS agus le OWL lena chéile mar teangacha bunaidh. Ar na samplaí den chur chuige seo tá Friend of a Friend (FOAF), ointeolaíocht shóisialta chun cur síos a dhéanamh ar dhaoine, ar a gcuid gníomhaíochtaí agus ar na gaolmhaireachtaí eatarthu agus Simple Knowledge Organisation System (SKOS), caighdeán eile chun eolas ar an nGréasán a eagrú.

${ }^{18} \mathrm{http}: / / w w w . w 3 . o r g / T R /$ rdf-schema/ 